\title{
Associations of impaired glucose metabolism and dyslipidemia with cardiovascular diseases: what have we learned from Japanese cohort studies for individualized prevention and treatment?
}

\author{
Yoshihiro Kokubo
}

Received: 12 January 2011 / Accepted: 10 March 2011 / Published online: 2 April 2011

(C) European Association for Predictive, Preventive and Personalised Medicine 2011

\begin{abstract}
Metabolic disorder is a modifiable risk factor for cardiovascular diseases (CVD), and lifestyle modification is the key to improving metabolic disorder. Diabetes mellitus has been shown to be a risk factor for coronary heart disease (CHD) and ischemic stroke in both Western and Japanese populations. An association between impaired fasting glucose and pre-hypertension found in an urban Japanese population emphasized the combined risk of CVD. Mean total cholesterol levels in Japan have been increasing in the last three decades. The Japanese evidence for the positive association of total cholesterol with CHD is similar to that in the West. Higher low-density lipoprotein cholesterol (LDL-C) levels pose an increased risk of $\mathrm{CHD}$ and atherothrombotic infarction, whereas lower LDL-C levels may pose an increased risk of intracerebral hemorrhage in Japan. Overall, the studies reviewed here show that impaired glucose metabolism and dyslipidemia are emerging risk factors for CVD in the Japanese population.
\end{abstract}

Keywords Impaired glucose metabolism · Dyslipidemia . Lifestyle $\cdot$ Predictors for cardiovascular disease $\cdot$ Cohort study · Japanese population

Y. Kokubo $(\bowtie)$

Department of Preventive Cardiology,

National Cerebral and Cardiovascular Center,

5-7-1, Fujishiro-dai,

Suita, Osaka 565-8565 Japan

e-mail: ykokubo@hsp.ncvc.go.jp

\section{Introduction}

Metabolic syndrome comprises a cluster of components of impaired glucose metabolism, abdominal fat accumulation, dyslipidemia, and elevated blood pressure [1]. Each component has been shown to be an independent risk factor for cardiovascular diseases (CVD) in Japanese community cohort studies: impaired fasting glucose [2]; abdominal obesity [3, 4]; low-density lipoprotein cholesterol $[5,6]$; and high-normal blood pressure [7-9].

Recently, a new streamlined definition of metabolic syndrome for global populations has been introduced [10]. In this definition, abdominal obesity is just one of the possible components for metabolic syndrome. However, in the Japanese definition of metabolic syndrome, abdominal obesity is an essential component [11]. The definition may mislead a prevention of CVD for non-obesity metabolic disorder, which is a modifiable risk factor for CVD. Changes in lifestyle are the key to improving metabolic disorder, including diabetes mellitus (DM) and dyslipidemia, and thereby to reducing the risk of cardiovascular disease. In this review paper, the focus is on the relation of metabolic symptoms, namely impaired glucose metabolism and dyslipidemia to CVD in the Japanese population.

Impaired glucose metabolism: trends, combination with blood pressure elevation, and individualized preventive approaches

Diabetes mellitus has become a major public health problem $[12,13]$ as well as a risk factor for mortality [12] and CVD 
[14-16]. In Japan, the frequencies of hyperglycemia for 1961, 1974, and 1989 show a trend of increasing incidence: $12.1 \%$, $13.8 \%$, and $31.9 \%$ in men and $4.8 \%, 8.1 \%$, and $27.2 \%$ in women, respectively [17].

Previous meta-analysis studies have shown that diabetes is a risk factor for ischemic stroke [18] and coronary artery disease [15, 18, 19]. The Framingham Offspring cohort study showed a positive relationship between impaired fasting glucose and coronary heart disease in women and between diabetes and coronary heart disease in men and women [20]. These results are compatible with those of previous cohort studies in Japan, as described below and summarized in Table 1.

The Hisayama Study indicated that diabetes as defined by the glucose tolerance test was a risk factor for ischemic stroke in men (hazard ratio 2.54; 95\% confidence intervals 1.40 to 4.63 ) and women (hazard ratio $2.02 ; 95 \%$ confidence intervals 1.07 to 3.81 ) and coronary heart disease in women (hazard ratio 3.46; 95\% confidence intervals 1.59 to 7.54) [21]; however, impaired glucose tolerance and impaired fasting glucose were not risk factors for ischemic stroke or coronary heart disease.

In a study of five communities in Japan, diabetes defined by non-fasting glucose levels was a risk factor for nonembolic ischemic stroke in men (hazard ratio 1.8; 95\% confidence intervals 1.0 to 3.2 ) and women (hazard ratio 2.2; $95 \%$ confidence intervals 1.2 to 4.0 ) [22]. This result is similar to that of lacunar infarction. The risk was observed in both non-hypertensive subjects and obese subjects. The positive association was particularly strong in hypertensive subjects with higher skin-fold thickness values (hazard ratio 1.9 and $95 \%$ confidence intervals 1.0 to 3.7 for borderline diabetes; hazard ratio 4.9 and $95 \%$ confidence intervals 2.5 to 9.5 for diabetes).

A positive association between diabetes and CVD mortality was also observed in a Japanese general population. The

Table 1 Association between blood glucose categories and cardiovascular diseases in Japanese cohort studies

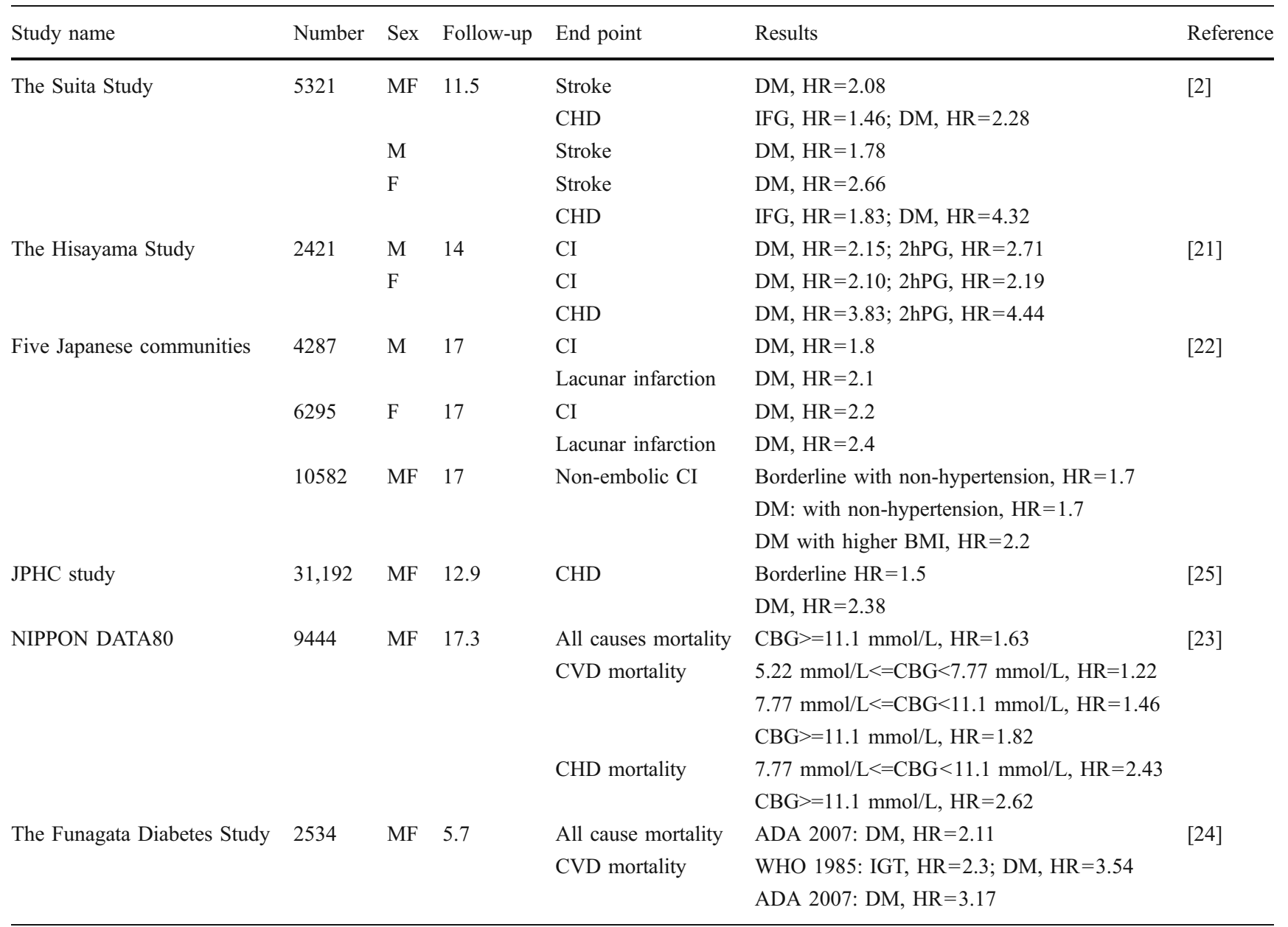

$M$ men; $F$ women; $C H D$ coronary heart disease; $C I$ cerebral infarction; $C V D$ cardiovascular diseases; $D M$ diabetes mellitus; $I F G$ impaired fasting glucose; $2 h P G 2 \mathrm{~h}$ post-loaded glucose levels; $H R$ hazard ratio; $B M I$ body mass index; $C B G$ casual blood glucose; $A D A$ American Diabetes Association; WHO World Health Organization. 
NIPPON DATA 80 Study indicated that high and borderlinehigh casual blood glucose groups $(11.1 \mathrm{mmol} / \mathrm{L}$ and 7.77 to $11.1 \mathrm{mmol} / \mathrm{L}$, respectively) had increased risks of coronary heart disease mortality [23]. Similar results were observed for both CVD and all-cause mortality. The Funagata Diabetes Study showed that diabetes defined by both the WHO criteria (1985) and ADA recommendations (1997) were risk factors for all-cause mortality and cardiovascular mortality [24]. However, impaired fasting glucose was not a risk factor for all-cause mortality and cardiovascular mortality. These Japanese cohort studies for mortality risks indicated that diabetes is a risk factor for all-cause and CVD mortality. However, impaired fasting glucose and impaired glucose tolerance may not be risk factors for all-cause or CVD mortality. Further investigations of larger cohort studies are required to clarify these matters.

Recently, the Suita Study showed that impaired fasting glucose is a risk factor for the incidence of cardiovascular disease (hazard ratio $1.49 ; 95 \%$ confidence intervals 1.02 to 2.16 ) or coronary heart disease (hazard ratio 1.83 ; $95 \%$ confidence intervals 1.01 to 3.32 ) in women, and that diabetes is a risk factor for stroke in both men (hazard ratio $1.78 ; 95 \%$ confidence intervals 1.00 to 3.12 ) and women (hazard ratio 2.66 ; $95 \%$ confidence intervals 1.22 to 5.80 ) and for coronary heart disease in women (hazard ratio 1.78; $95 \%$ confidence intervals 1.00 to 3.12 ) [2]. In addition, compared with normoglycemic and optimal blood pressure Japanese subjects, increased risks of CVD were observed in normoglycemic subjects with high-normal blood pressure or hypertension, impaired fasting glucose subjects with normal or higher blood pressure, and diabetic subjects regardless of blood pressure category (Fig. 1: P-value for interaction $=0.046$ ). These two borderline categories may augment the risk of CVD.

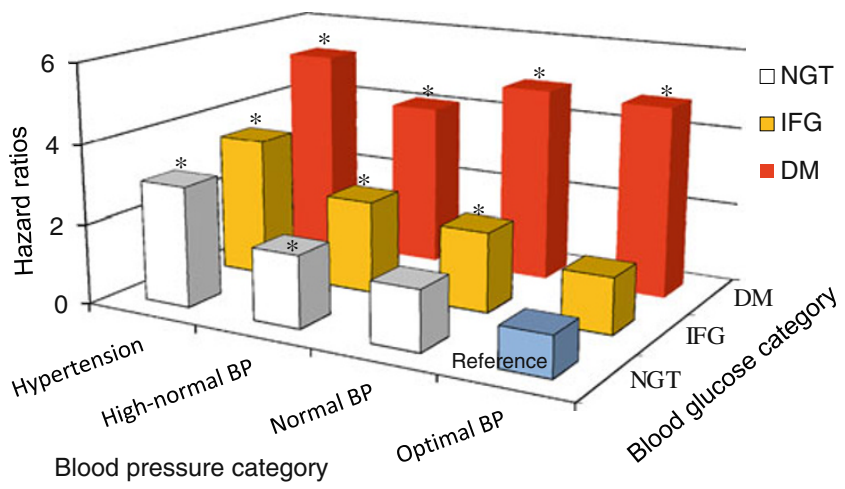

Fig. 1 Multivariable-adjusted hazard ratios of cardiovascular diseases according to the combination of blood pressure and glucose categories. Multivariable-adjusted hazard ratios were adjusting for age, sex, body mass index, smoking, drinking, and hyperlipidemia. $B P$ blood pressure; NGT normal glycemic tolerance; IFG impaired fasting glucose; $D M$ diabetes mellitus; *: $P<0.05$ (compared with the optimal blood pressure and normoglycemic group)
In a collaborative meta-analysis of 102 prospective studies from around the world, increased risks of coronary heart disease were observed in unknown diabetic subjects (i.e., subjects with diabetes who are not aware of their disease) with 5.6 to $<6.1 \mathrm{mmol} / \mathrm{L}$ and 6.1 to $<7 \mathrm{mmol} / \mathrm{L}$ (hazard ratios [95\% confidence intervals]: 1.11 [1.04 to 1.18] and 1.17 [1.08 to 1.26], respectively) [18]. Quite recently, the Japan Public Health Center-based prospective (JPHC) study consisting of a 12.9-year of follow-up for 31,192 individuals aged 40-69 years was observed that diabetes mellitus and hyperglycemia carried an increased risk for coronary heart disease in a Japanese general population [25]. Based on this study and the Suita study $[2,25]$, both diabetes mellitus and impaired fasting glucose may be a risk factor for the incidence of coronary heart disease in Japan.

In the JPHC study's systematic review of the evidence for pre-diabetes, impaired fasting glucose and impaired glucose tolerance were shown to be associated with modest increases in the risk for CVD [25]. Overall relative risks ( $95 \%$ confidence intervals) for the association between two categories of impaired fasting glucose (100 to $125 \mathrm{mg} / \mathrm{dL}$ and 110 to $125 \mathrm{mg} / \mathrm{dL}$ ) and CVD were 1.18 (1.09 to 1.28$)$ and 1.20 (1.12 to 1.28 ), respectively. Meanwhile, the risks of CVD for impaired fasting glucose and impaired glucose tolerance were 1.10 (0.98 to 1.23 ) and 1.20 (1.07 to 1.34 ), respectively. In two Japanese cohort studies using the oral glucose tolerance test, impaired fasting glucose was not found to pose a risk of CVD [21, 24]. However, in one of these cohort studies, impaired glucose tolerance was observed as a risk of CVD mortality [24]. Larger Japanese cohort studies are required to establish the association between oral glucose tolerance test results and CVD.

In order to prevent CVD, a person with impaired glucose metabolism should eliminate or reduce other cardiovascular risk factors, such as high blood pressure, obesity, smoking, and excess drinking. The Suita study showed that the combination of impaired fasting glucose and high blood pressure greatly increased the risk of cardiovascular disease [2]. Recently, the frequencies of obesity in men and elderly women have increased in Japan [26]. Obesity is a risk factor for impaired glucose metabolism; weight loss reduces the risk of both impaired glucose metabolism [27] and CVD [3].

The average smoking rate in men worldwide has rapidly decreased in recent years, and in 2005 had dropped by $45.5 \%$ since its peak. However, the rate in Japan is still higher than that in Western countries [28]. And while, the smoking rate in women is generally lower than that in men. In Japan, the rate for younger women aged 20 to 39 years has increased to around 17 to $20 \%$ [28]. Smoking itself is a risk factor for diabetes mellitus [29]. Smoking cessation reduces the risk of both diabetes mellitus [30] and CVD 
[31]. Excess drinking is a risk factor for increasing blood pressure [32] and stroke [33]. In order to reduce the risk of CVD, subjects with a combination of two borderline risks, high-normal blood pressure and hyperglycemia, should modify their lifestyles, aiming at cessation of smoking and even moderate drinking, as well as weight reduction if there are overweight or obese.

\section{Dyslipidemia: risks, trends, and individual treatment approaches}

According to Japan's National Survey on Circulatory Disorders in 1980, 1990, and 2000, mean levels of total cholesterol increased from $186 \mathrm{mg} / \mathrm{dL}$ and $191 \mathrm{mg} / \mathrm{dL}$ in 1980 to $200 \mathrm{mg} / \mathrm{dL}$ and $208 \mathrm{mg} / \mathrm{dL}$ in 2000 among adult men and women, respectively. Frequencies of hypercholesterolemia (total cholesterol levels of $220 \mathrm{mg} / \mathrm{dL}$ or more) increased $15 \%$ and $19 \%$ in 1980 to $27 \%$ and $35 \%$ in 2000 among men and women, respectively [34]. The difference in mean total cholesterol levels between Japanese and American men was approximately $40 \mathrm{mg} / \mathrm{dL}$ in the $1980 \mathrm{~s}$. However, this difference had diminished to $15 \mathrm{mg} / \mathrm{dL}$ by 1990 and 2000. No changes in mean total cholesterol levels in Japanese or American men aged 60 years and over were observed from 1990 to 2000 [28].

\section{Total cholesterol}

A previous meta-analytic study has shown that total cholesterol was positively associated with ischemic heart disease in both middle and old age and at all blood pressure categories [35]. In contrast, the association between total cholesterol and the risk of stroke remains unclear. The Multiple Risk Factor Intervention trial has shown that the risk of death from intracerebral hemorrhage was three times higher in men with serum cholesterol levels under $160 \mathrm{mg} / \mathrm{dL}$ than in those with higher cholesterol levels, whereas a positive association was observed between the serum cholesterol level and death from ischemic stroke [36]. A meta-analysis of 45 prospective cohort studies showed no association between blood cholesterol levels and stroke except in those under 45 years of age when screened [37]. The inconsistency in results may be due to the different etiologic origins of stroke. Dyslipidemia may be important for some subtypes of stroke but not for others, because stroke is a heterogeneous disease of various etiologic origins. In American men, an inverse association was observed between the total cholesterol levels and the incidence of intracerebral hemorrhage, while a positive association was observed between total cholesterol levels and the incidence of cerebral infarction [36].
Japanese cohort studies have shown evidence of the positive association of total cholesterol with coronary heart disease similar to that in Western studies. NIPPON DATA80 showed associations between total cholesterol and risk of all-cause mortality (hazard ratio 1.19 and 1.36; $95 \%$ confidence intervals, 1.03 to 1.37 and 1.05 to 1.77 ) in both the lowest $(<160 \mathrm{mg} / \mathrm{dl})$ and highest $(\geq 260 \mathrm{mg} / \mathrm{dl})$ total cholesterol groups, respectively [38]. In addition, the hazard ratios of coronary heart disease mortality were 1.4 in the 200 to $219 \mathrm{mg} / \mathrm{dL}$ total cholesterol group, 1.7 in the 220 to $239 \mathrm{mg} / \mathrm{dL}$ group, 1.8 in the 240 to $259 \mathrm{mg} / \mathrm{dL}$ groups, and 3.8 in the $260 \mathrm{mg} / \mathrm{dL}$ or above group, compared with the 160 to $179 \mathrm{mg} / \mathrm{dL}$ total cholesterol (healthy control) group [38].

\section{LDL cholesterol and non-HDL cholesterol}

The Suita Study showed a positive association between serum low-density lipoprotein (LDL) cholesterol and nonhigh-density lipoprotein (non-HDL) cholesterol levels and increased incidence of myocardial infarction, but not with any type of stroke [6]. The hazard ratio for myocardial infarction was highest in the top quintile of LDL cholesterol (hazard ratio, 3.03; 95\% confidence intervals, 1.32 to 6.96) when men and women were combined. The hazard ratio for myocardial infarction was also highest in the top quintile of non-HDL cholesterol (hazard ratio, 2.97; 95\% confidence intervals, 1.26 to 6.97 ). There was no substantial difference in the predictive value for the incidence of myocardial infarction between LDL cholesterol and non-HDL cholesterol. LDL cholesterol can be calculated from fasting blood sample and measurement of total cholesterol, HDL cholesterol, and triglyceride levels from fasting blood samples, according to the Friedewald formula [39]. The formula is not applicable for serum triglyceride levels equal to or greater than $\geq 400 \mathrm{mg} / \mathrm{dL}$. However, non-HDL cholesterol levels can be easily calculated by routine measured parameters, total and HDL cholesterol without the effect of non-fasting status or hypertriglyceridemia $(\geq 400 \mathrm{mg} / \mathrm{dL})$.

The Hisayama Study showed that the positive association between LDL cholesterol levels and risk of atherothrombotic infarction $(\mathrm{P}$ for trend $=0.02)$ and coronary heart disease $(\mathrm{P}$ for trend $=0.03$ ) remained significant after multivariable adjustment. In the Ibaraki Prefectural Health Study, which was a very large sample consisting of 30,802 men and 60,417 women, high LDL cholesterol levels were associated with an increased risk of mortality from coronary heart disease in men, but not in women [40]. The same study showed that lower LDL cholesterol levels are associated with elevated risk of mortality from intracerebral hemorrhage, but are not associated with increasing risk of cerebral infarction [41]. These Japanese cohort studies suggest that higher levels 
of LDL cholesterol pose an increased risk of coronary heart disease and possibly atherothrombotic infarction, whereas lower levels of LDL cholesterol may increase the risk of intracerebral hemorrhage.

Recently, the lectin-like oxidized LDL receptor 1 (LOX-1) has been implicated in atherothrombotic diseases [42]. Activation of LOX-1 in humans can be evaluated by use of the LOX index, obtained by multiplying the circulating concentration of LOX-1 ligands containing apolipoprotein B by that of the soluble form of LOX-1. In the Suita Study, higher LOX index values were associated with an increased risk of coronary heart disease [43].

HDL cholesterol

Lower HDL cholesterol predicts coronary heart disease mortality and occurrence of new coronary heart disease events [44]. Elevated total cholesterol was not found to be associated with coronary heart disease mortality in older men, but may be a risk factor for coronary heart disease in older women [44]. The Israeli Ischemic Heart Disease Study showed an independent negative association between HDL cholesterol and ischemic stroke mortality during 21 years of follow-up [45].

Among the Japanese cohort studies, the Oyabe Study demonstrated that lower HDL cholesterol levels were related significantly and independently to an increased risk of all-stroke incidence and ischemic stroke incidence [46]. And in a combined prospective cohort study of 13 urban industrial companies, coronary heart disease incidence was inversely related to HDL cholesterol in Japanese men [47].

\section{Triglyceride}

Based on combined data from prospective studies, a high serum triglyceride level is a risk factor for CVD for both men (hazard ratio, $1.32 ; 95 \%$ confidence intervals, 1.26 to 1.39 ) and women (hazard ratio, 1.76; 95\% confidence intervals, 1.50 to 2.07 ) in the general population, independent of HDL cholesterol [48]. In 26 prospective studies in Asian and Pacific populations, serum triglycerides were an independent predictor of coronary heart disease and stroke risk [49]. In 29 prospective meta-analytic studies in the West, triglyceride levels were moderately associated with coronary heart disease [50].

In Japanese cohort studies, two studies have provided evidence regarding the association between serum triglyceride levels and coronary heart disease and ischemic stroke. A cohort study of four rural communities showed that a high serum triglyceride level was a risk factor for coronary heart disease; even after adjustment for HDL cholesterol levels the significant association remained, although $80 \%$ of the baseline participants were non-fasting [51]. The Suita Study has also shown that the risk for ischemic stroke was highest in participants with high triglycerides alone, and that a combination of high serum levels of triglyceride and nonHDL cholesterol was associated with an increased risk of myocardial infarction [52]. High serum levels of triglyceride and non-HDL cholesterol are both important targets for the prevention of CVD, which requires evidence-based guidelines for management in the primary care setting.

\section{Concluding remarks and outlook}

This paper reviews the associations of impaired glucose metabolism and dyslipidemia with CVD in Japanese cohort studies. Diabetes mellitus is a risk factor for coronary heart disease and ischemic stroke. Impaired fasting glucose and high-normal blood pressure were shown to be independent risk factors for CVD and coronary heart disease in an urban cohort. The combination of these two borderline categories may increase the risk for CVD. Impaired glucose tolerance has not been observed as a risk factor for the incidence of CVD in Japan. The Japanese evidence for the positive association of total cholesterol with coronary heart disease is similar to that of previous Western studies. Associations with all-cause mortality were observed for both the lower and higher levels of cholesterol: Higher levels of LDL cholesterol have been shown to increase the risk of coronary heart disease and atherothrombotic infarction, whereas lower levels of LDL cholesterol may increase the risk of intracerebral hemorrhage in Japan, as elsewhere. HDL cholesterol levels were inversely related with ischemic stroke. Positive associations between serum triglyceride levels and coronary heart disease and ischemic stroke have also been observed in Japanese populations. The Japanese diet has been rapidly changing in recent decades, as reflected in many of its health indicators such as cholesterol levels, and both impaired glucose metabolism and dyslipidemia are emerging as important risk factors for CVD in the Japanese population. In order to reduce the risk of CVD, subjects with metabolic disorder should reduce other cardiovascular risk factors and improve their lifestyle.

\section{References}

1. Eckel RH, Grundy SM, Zimmet PZ. The metabolic syndrome. Lancet. 2005;365:1415-28.

2. Kokubo Y, Okamura T, Watanabe M, et al. The combined impact of blood pressure category and glucose abnormality on the incidence of cardiovascular diseases in a Japanese urban cohort: the Suita Study. Hypertens Res. 2010;33:1238-43.

3. Furukawa Y, Kokubo Y, Okamura T, et al. The relationship between waist circumference and the risk of stroke and myocar- 
dial infarction in a Japanese urban cohort: the Suita study. Stroke. 2010;41:550-3.

4. Doi Y, Ninomiya T, Hata J, et al. Proposed criteria for metabolic syndrome in Japanese based on prospective evidence: the Hisayama study. Stroke. 2009;40:1187-94.

5. Executive Summary of the Third Report of the National Cholesterol Education Program (NCEP). Expert panel on detection, evaluation, and treatment of high blood cholesterol in adults (Adult Treatment Panel III). JAMA. 2001;285:2486-97.

6. Okamura T, Kokubo Y, Watanabe M, et al. Low-density lipoprotein cholesterol and non-high-density lipoprotein cholesterol and the incidence of cardiovascular disease in an urban Japanese cohort study: The Suita study. Atherosclerosis. 2009;203:587-92.

7. Kokubo Y, Kamide K, Okamura T, et al. Impact of high-normal blood pressure on the risk of cardiovascular disease in a Japanese urban cohort: the Suita study. Hypertension. 2008;52:652-9.

8. Ikeda A, Iso H, Yamagishi K, Inoue M, Tsugane S. Blood pressure and the risk of stroke, cardiovascular disease, and all-cause mortality among Japanese: the JPHC Study. Am J Hypertens. 2009;22:273-80.

9. Kokubo Y, Kamide K. High-normal blood pressure and the risk of cardiovascular disease. Circ J. 2009;73:1381-5.

10. Alberti KG, Eckel RH, Grundy SM, et al. Harmonizing the metabolic syndrome: a joint interim statement of the international diabetes federation task force on epidemiology and prevention; national heart, lung, and blood institute; american heart association; world heart federation; international atherosclerosis society; and international association for the study of obesity. Circulation. 2009;120:1640-5.

11. Kokubo Y, Okamura T, Yoshimasa Y, et al. Impact of metabolic syndrome components on the incidence of cardiovascular disease in a general urban Japanese population: the Suita study. Hypertens Res. 2008;31:2027-35.

12. Garcia MJ, McNamara PM, Gordon T, Kannel WB. Morbidity and mortality in diabetics in the Framingham population. Sixteen year follow-up study. Diabetes. 1974;23:105-11.

13. Manuel DG, Schultz SE. Health-related quality of life and healthadjusted life expectancy of people with diabetes in Ontario, Canada, 1996-1997. Diabetes Care. 2004;27:407-14.

14. Fox CS, Coady S, Sorlie PD, et al. Increasing cardiovascular disease burden due to diabetes mellitus: the framingham heart study. Circulation. 2007;115:1544-50.

15. Lee WL, Cheung AM, Cape D, Zinman B. Impact of diabetes on coronary artery disease in women and men: a meta-analysis of prospective studies. Diabetes Care. 2000;23:962-8.

16. Folsom AR, Rasmussen ML, Chambless LE, et al. Prospective associations of fasting insulin, body fat distribution, and diabetes with risk of ischemic stroke. The Atherosclerosis Risk in Communities (ARIC) study investigators. Diabetes Care. 1999;22:1077-83.

17. Kubo M, Kiyohara Y, Kato I, et al. Trends in the incidence, mortality, and survival rate of cardiovascular disease in a Japanese community: the Hisayama study. Stroke. 2003;34:2349-54.

18. Sarwar N, Gao P, Seshasai SR, et al. Diabetes mellitus, fasting blood glucose concentration, and risk of vascular disease: a collaborative meta-analysis of 102 prospective studies. Lancet. 2010;375:2215-22.

19. Sarwar N, Aspelund T, Eiriksdottir G, et al. Markers of dysglycaemia and risk of coronary heart disease in people without diabetes: Reykjavik prospective study and systematic review. PLoS Med. 2010;7:e1000278.

20. Levitzky YS, Pencina MJ, D'Agostino RB, et al. Impact of impaired fasting glucose on cardiovascular disease: the framingham heart study. J Am Coll Cardiol. 2008;51:264-70.

21. Doi Y, Ninomiya T, Hata J, et al. Impact of glucose tolerance status on development of ischemic stroke and coronary heart disease in a general Japanese population: the Hisayama study. Stroke. 2010;41:203-9.

22. Iso $\mathrm{H}$, Imano $\mathrm{H}$, Kitamura $\mathrm{A}$, et al. Type 2 diabetes and risk of non-embolic ischaemic stroke in Japanese men and women. Diabetologia. 2004;47:2137-44.

23. Kadowaki S, Okamura T, Hozawa A, et al. Relationship of elevated casual blood glucose level with coronary heart disease, cardiovascular disease and all-cause mortality in a representative sample of the Japanese population. NIPPON DATA80. Diabetologia. 2008;51:575-82.

24. Tominaga M, Eguchi H, Manaka H, Igarashi K, Kato T, Sekikawa A. Impaired glucose tolerance is a risk factor for cardiovascular disease, but not impaired fasting glucose. The Funagata Diabetes Study. Diabetes Care. 1999;22:920-4.

25. Saito I, Kokubo Y, Yamagishi K, Iso H, Inoue M, Tsugane $\mathrm{S}$. Diabetes and the risk of coronary heart disease in the general Japanese population: the Japan Public Health Center-based prospective (JPHC) study. Atherosclerosis 2011. doi:10.1016/j. atherosclerosis.2011.01.021.

26. Yoshiike N, Seino F, Tajima S, et al. Twenty-year changes in the prevalence of overweight in Japanese adults: the national nutrition survey 1976-95. Obes Rev. 2002;3:183-90.

27. Lechleitner M. Obesity and the metabolic syndrome in the elderly-a mini-review. Gerontology. 2008;54:253-9.

28. Ueshima H. Explanation for the Japanese paradox: prevention of increase in coronary heart disease and reduction in stroke. $\mathrm{J}$ Atheroscler Thromb. 2007;14:278-86.

29. Willi C, Bodenmann P, Ghali WA, Faris PD, Cornuz J. Active smoking and the risk of type 2 diabetes: a systematic review and meta-analysis. JAMA. 2007;298:2654-64.

30. Yeh HC, Duncan BB, Schmidt MI, Wang NY, Brancati FL. Smoking, smoking cessation, and risk for type 2 diabetes mellitus: a cohort study. Ann Intern Med. 2010;152:10-7.

31. Honjo K, Iso $\mathrm{H}$, Tsugane $\mathrm{S}$, et al. The effects of smoking and smoking cessation on mortality from cardiovascular disease among Japanese: pooled analysis of three large-scale cohort studies in Japan. Tob Control. 2010;19:50-7.

32. Beilin LJ, Puddey IB, Burke V. Alcohol and hypertension-kill or cure? J Hum Hypertens. 1996;10 Suppl 2:S1-5.

33. Iso $\mathrm{H}, \mathrm{Baba} \mathrm{S}$, Mannami $\mathrm{T}$, et al. Alcohol consumption and risk of stroke among middle-aged men: the JPHC study cohort I. Stroke. 2004;35:1124-9.

34. Iso H. Changes in coronary heart disease risk among Japanese. Circulation. 2008;118:2725-9.

35. Lewington S, Whitlock G, Clarke R, et al. Blood cholesterol and vascular mortality by age, sex, and blood pressure: a metaanalysis of individual data from 61 prospective studies with 55,000 vascular deaths. Lancet. 2007;370:1829-39.

36. Iso H, Jacobs Jr DR, Wentworth D, Neaton JD, Cohen JD. Serum cholesterol levels and six-year mortality from stroke in 350,977 men screened for the multiple risk factor intervention trial. N Engl J Med. 1989;320:904-10.

37. Cholesterol, diastolic blood pressure, and stroke: 13,000 strokes in 450,000 people in 45 prospective cohorts. Prospective studies collaboration. Lancet. 1995;346:1647-53.

38. Okamura T, Tanaka H, Miyamatsu N, et al. The relationship between serum total cholesterol and all-cause or cause-specific mortality in a 17.3-year study of a Japanese cohort. Atherosclerosis. 2007;190:216-23.

39. Friedewald WT, Levy RI, Fredrickson DS. Estimation of the concentration of low-density lipoprotein cholesterol in plasma, without use of the preparative ultracentrifuge. Clin Chem. 1972;18:499-502.

40. Noda H, Iso H, Irie F, Sairenchi T, Ohtaka E, Ohta H. Gender difference of association between LDL cholesterol concentra- 
tions and mortality from coronary heart disease amongst Japanese: the Ibaraki prefectural health study. J Intern Med. 2010;267:576-87.

41. Noda H, Iso H, Irie F, et al. Low-density lipoprotein cholesterol concentrations and death due to intraparenchymal hemorrhage: the Ibaraki prefectural health study. Circulation. 2009;119:2136-45.

42. Sawamura T, Kume N, Aoyama T, et al. An endothelial receptor for oxidized low-density lipoprotein. Nature. 1997;386:73-7.

43. Inoue $\mathrm{N}$, Okamura $\mathrm{T}$, Kokubo $\mathrm{Y}$, et al. LOX index, a novel predictive biochemical marker for coronary heart disease and stroke. Clin Chem. 2010;56:550-8.

44. Corti MC, Guralnik JM, Salive ME, et al. HDL cholesterol predicts coronary heart disease mortality in older persons. JAMA. 1995;274:539-44.

45. Goldbourt U, Yaari S, Medalie JH. Isolated low HDL cholesterol as a risk factor for coronary heart disease mortality. A 21-year follow-up of 8000 men. Arterioscler Thromb Vasc Biol. 1997; 17:107-13.

46. Soyama Y, Miura K, Morikawa Y, et al. High-density lipoprotein cholesterol and risk of stroke in Japanese men and women: the Oyabe Study. Stroke. 2003;34:863-8.
47. Kitamura A, Iso $H$, Naito $Y$, et al. High-density lipoprotein cholesterol and premature coronary heart disease in urban Japanese men. Circulation. 1994;89:2533-9.

48. Hokanson JE, Austin MA. Plasma triglyceride level is a risk factor for cardiovascular disease independent of high-density lipoprotein cholesterol level: a meta-analysis of population-based prospective studies. J Cardiovasc Risk. 1996;3:213-9.

49. Patel A, Barzi F, Jamrozik K, et al. Serum triglycerides as a risk factor for cardiovascular diseases in the Asia-Pacific region. Circulation. 2004;110:2678-86.

50. Sarwar N, Danesh J, Eiriksdottir G, et al. Triglycerides and the risk of coronary heart disease: 10,158 incident cases among 262,525 participants in 29 Western prospective studies. Circulation. 2007;115:450-8.

51. Iso $\mathrm{H}$, Naito $\mathrm{Y}$, Sato $\mathrm{S}$, et al. Serum triglycerides and risk of coronary heart disease among Japanese men and women. Am J Epidemiol. 2001;153:490-9.

52. Okamura T, Kokubo Y, Watanabe M, et al. Triglycerides and nonhigh-density lipoprotein cholesterol and the incidence of cardiovascular disease in an urban Japanese cohort: the Suita study. Atherosclerosis. 2010;209:290-4. 